community health and participation in research (Table 4). This work and the data presented here are important for informing CEnR approaches and will be useful for guiding the development of a model incorporating the core tenets of CEnR within the mission, vision, and priorities of aLHSs.

\section{Understanding Racial Disparities in Hepatocellular Carcinoma Treatments and Outcomes}

Lauren Devore Nephew ${ }^{1}$, Susan Rawl and Naga Chalasani

${ }^{1}$ Indiana Univeristy School of Medicine

OBJECTIVES/SPECIFIC AIMS: Black patients with hepatocellular carcinoma (HCC) receive fewer curative therapies and have higher mortality than other groups. Reducing this disparity will require an in-depth understanding of patient comorbidities, tumor characteristics, and social determinants of health. Our objectives are to a) perform a multi-center retrospective cohort study of black and white patients diagnosed with HCC in the Indianapolis area. b) prospectively enroll black and white patients with HCC to collect clinical characteristics as well as data on the social determinants of health. METHODS/STUDY POPULATION: A retrospective chart review of patients with a diagnosis of HCC from 2010-2017 from five area Indianapolis hospitals will be performed. Demographics, comorbidities, liver disease severity, and tumor characteristics will be collected using the Indiana Network for Patient Care database and compared between black and white patients. Concomitantly, a prospective cohort of black and white patients will be enrolled and surveyed to collect data on socioeconomic status and income adequacy, literacy, functional status, substance abuse history, social support, activation, and adherence. The primary outcomes are the receipt of curative therapies for HCC including liver transplantation, resection or ablation. The secondary outcome is mortality. Multivariable logistic regression models will be used to explore disparities seen in the primary and secondary outcomes. RESULTS/ ANTICIPATED RESULTS: These preliminary results include Indiana University Hospital (IUH) findings; a multicenter analysis is underway. The IUH cohort included 1,032 (86\%) white and 164 (14\%) black patients. Black and white patients had similar Model for End-Stage Liver Disease and Child-Pugh scores. There was a trend toward larger tumor size $(5.3 \mathrm{~cm}$ vs. $4.7 \mathrm{~cm} ; \mathrm{P}=0.05)$ in black patients; however, Barcelona Clinic Liver Cancer staging and Milan criteria were similar. Black patients were less likely to undergo liver transplantation than white patients-a disparity that was not attenuated (odds ratio $[\mathrm{OR}], 0.43 ; 95 \%$ confidence interval [CI]: 0.21-0.90) on multivariable analysis. Substance abuse was more frequently cited as the reason black patients within Milan criteria failed to undergo transplantation than white patients. Survival was similar between the two groups. DISCUSSION/ SIGNIFICANCE OF IMPACT: Racial differences in patient and tumor characteristics were small in our single center analysis and did not explain the disparity in liver transplantation. This analysis however only reflects $25 \%$ of patients diagnosed with HCC in the Indianapolis metropolitan, highlighting the need for a multicenter study. Higher rates of substance abuse in black patients within Milan criteria who failed to undergo transplantation suggest social factors contribute to this disparity and highlight the need for a prospective study that can explore these and other social factors.

3406

Understanding the Barriers, Challenges, and Facilitators to Community-Engaged Research: A Review of a CTSA Community Engagement Pilot Program

Ashley Dunn ${ }^{1}$, Kendra L. Smith, Rhonda McClinton-Brown, Jill W. Evans, Lisa Goldman-Rosas and Mark Cullen

${ }^{1}$ Stanford University School of Medicine

OBJECTIVES/SPECIFIC AIMS: Engaging patients and consumers in research is a complex process where innovative strategies are needed to effectively translate scientific discoveries into improvements in the public's health (Wilkins et. al., 2013; Terry et. al., 2013). The Clinical Translational Science Awards (CTSA)supported by the National Institute of Health $(\mathrm{NIH})$ under the auspices of the National Center for Advancing Translational Sciences (NCATS) - aim to provide resources and support needed to strengthen our nation's clinical and translational research (CTR) enterprise. In 2008, Stanford University was awarded a CTSA from the NIH, establishing Spectrum (Stanford Center for Clinical and Translational Research and Education) and its Community Engagement (CE) Program aimed at building long-standing community-academic research partnerships for translational research in the local area surrounding Stanford University. To date, the CE Pilot Program has funded 38 pilot projects from the 2009-2017 calendar year. The purpose of this study was to understand, through a unique pilot program, the barriers, challenges, and facilitators to community-engaged research targeting health disparities as well as community-academic partnerships. METHODS/STUDY POPULATION: Investigators conducted a qualitative study of the community engagement pilot program. Previous pilot awardees were recruited via email and phone to participate in a one-hour focus group to discuss their pilot project experience-describing any barriers, challenges, and facilitators to implementing their pilot project. RESULTS/ANTICIPATED RESULTS: The focus group revealed that community engage research through the pilot program was not only appreciated by faculty, but projects were successful, and partnerships developed were sustained after funding. Specifically, the pilot program has seen success in both traditional and capacity building metrics: the initial investment of $\$ 652,250.00$ to fund 38 projects has led to over $\$ 11$ million dollars in additional grant funding. In addition, pilot funding has led to peer-reviewed publications, data resources for theses and dissertations, local and national presentations/news articles, programmatic innovation, and community-level impact. Challenges and barriers were mainly related to timing, grant constraints, and university administrative processes. DISCUSSION/SIGNIFICANCE OF IMPACT: The Community Engagement Pilot Program demonstrates an innovative collaborative approach to support community-academic partnerships. This assessment highlights the value and importance of pilot program to increase community engaged research targeting health disparities. Challenges are mainly administrative in nature: pilot awardees mentioned difficulties working on university quarterly timelines, challenges of subcontracting or sharing money with community partners, onerous NIH prior 